\title{
Platelet Membrane Defects in Glanzmann's
}

\section{Thrombasthenia}

\section{EVIDENCE FOR DECREASED AMOUNTS OF TWO MAJOR GLYCOPROTEINS}

\author{
David R. Phillips and Patricia Poh Agin \\ From the Department of Biochemistry, St. Jude Children's Research Hospital, Memphis, Tennessee \\ 38101
}

A B S T RACT Platelets from patients with Glanzmann's thrombasthenia have a distinct molecular alteration of the plasma membrane surface, namely decreased amounts of a major glycoprotein designated as IIb (apparent mol wt 142,000). To identify other possible surface defects of thrombasthenic platelets, we labeled the membrane polypeptides of normal and thrombasthenic platelets by two different techniques: lactoperoxidase-catalyzed iodination and galactose oxidase oxidation, followed by reduction with tritiated sodium borohydride. Labeling patterns were determined after the polypeptides were separated by twodimensional polyacrylamide gel electrophoresis. Before the second dimension was run, platelet samples were incubated with a reducing agent, $\beta$-mercaptoethanol, to cleave the disulfide bonds of certain glycoproteins; the resulting changes in electrophoretic mobility permitted better resolution of individual molecules. Comparison of the labeled polypeptides of normal and thrombasthenic samples after reduction indicated decreased labeling of two major glycoproteins in thrombasthenic platelets: IIb and III (apparent mol wt 114,000 ). The relative proportions of radioactivity incorporated by these polypeptides were about 60 and $80 \%$ less than control values, respectively. With either Coomassie Blue or periodic acidSchiff's reagent, glycoprotein III stained much less intensely in thrombasthenic compared to normal samples, indicating that the observed labeling deficit was caused by a decreased concentration of the molecule rather than steric inaccessibility on the membrane surface. Analysis of normal plasma membranes by af-

Dr. Phillips is a recipient of a Research Career Development Award, HL-0800, from the National Heart and Lung Institute.

Received for publication 3 June 1976 and in revised form 1 April 1977. finity chromatography showed that glycoprotein IIb has receptors for lectin from Lens culinaris, the common lentil, whereas' III does not. We conclude that a characteristic feature of Glanzmann's thrombasthenia is a decreased concentration of two discrete glycoproteins in the platelet plasma membrane.

\section{INTRODUCTION}

Glanzmann's thrombasthenia, an inherited disorder of blood platelets, is characterized by a prolonged bleeding time, abnormal clot retraction, a normal platelet count, and the absence of platelet aggregation $(1,2)$. Although certain enzymatic differences have been reported for thrombasthenic vs. normal platelets (3-5), the molecular basis of the disorder appears to be an altered membrane surface, as suggested by several lines of evidence (6-9).

The first convincing demonstration of a specific membrane defect on thrombasthenic platelets came from the work of Nurden and Caen $(10,11)$. Using polyacrylamide disc-gel electrophoresis to separate membrane polypeptides and glycoproteins, and periodic acid-Schiff's (PAS) ${ }^{1}$ reagent to stain carbohydrate, they could not detect a major PAS-positive glycoprotein and concluded that its absence was a distinguishing feature of thrombasthenia. More recent studies (12) have established that this glycoprotein, now termed

\footnotetext{
${ }^{1}$ Nomenclature and abbreviations used in this paper: Our notation system for glycoproteins follows the current convention $(13,14)$, in which Roman numeral I designates the molecule with the highest molecular weight, Roman numeral II the next highest and so on. The letters $a, b$, and c denote discrete molecular species within molecularweight classes, and subscripts $\alpha$ and $\beta$ denote the subunits (alpha or beta chains) of individual glycoproteins. DOC, sodium deoxycholate; PAS, periodic acid-Schiff's reagent; SDS, sodium dodecyl sulfate.
} 
IIb, is indeed altered in thrombasthenic platelets, but that the alteration is a decrease in concentration rather than a complete absence of the molecule.

Analyzing the plasma membranes of thrombasthenic and normal platelets for differences in polypeptide composition is complicated by the presence of numerous proteins and glycoproteins, some with nearly identical molecular weights (13). Further, surface proteins may be altered during the preparation of platelets for electrophoresis, and some do not stain well enough to be clearly identified. Lactoperoxidase-catalyzed iodination of membrane polypeptides, followed by two-dimensional electrophoresis of nonreduced-reduced samples, circumvents these problems and permits a more comprehensive analysis of plasma membrane composition (13). These procedures were therefore adopted for further investigation of the exposed proteins of thrombasthenic platelets. Reported here is evidence that Glanzmann's thrombasthenia is characterized by decreased concentrations of two different membrane glycoproteins: IIb and another major glycoprotein, termed III.

\section{METHODS}

Clinical material. Platelets from six normal donors and from five patients who fulfilled the diagnostic criteria of Glanzmann's thrombasthenia $(1,2)$ were studied. Two patients were sisters; another sister in the same family also had thrombasthenia, and her platelets were the basis of an earlier report (9). The remaining three patients, two brothers and a sister, were from a different family and all have been subjects in previous studies (15-17). All experiments were repeated twice for each patient; an exception was two-dimensional electrophoresis, in which three platelet samples were used, two from the first family and one from the second. The relative proportions of label incorporated by glycoproteins did not vary from patient to patient, so only representative results are presented in autoradiograms and fluorograms.

Chemicals. Carrier-free $\mathrm{Na}{ }^{125} \mathrm{I}$, sp act $16 \mathrm{Ci} / \mathrm{mg}$, was purchased from Schwarz/Mann Div., Becton, Dickonson \& Co., Orangeburg, N. Y.; and tritiated sodium borohydride, sp act $15 \mathrm{Ci} / \mathrm{mmol}$, from Research Products International Corp., Elk Grove Village, Ill. Sources of other chemicals were: galactose oxidase (lot no. GAO 56K390) and $\beta$ galactosidase, Worthington Biochemical Corp., Freehold, N. J.; neuraminidase (lot no. BZ 2497), Schwarz/Mann Div.; bovine serum albumin, ovalbumin, azocasein, and $\alpha$-methylD-mannoside, Sigma Chemical Co., St. Louis, Mo.; chymotrypsinogen A, Mann Research Laboratories, New York; and Moni-Trol, American Hospital Supply Corp., Miami Fla. The galactose oxidase (62 U/mg of protein) was found to be heavily contaminated with proteolytic enzymes and was further purified by the method of Hatton and Regoeczi (18) to a specific activity of $830 \mathrm{U} / \mathrm{mg}$ of protein. It was treated with phenylmethylsulfonyl floride (Sigma Chemical Co.), $280 \mu \mathrm{g} / \mathrm{ml}$, which inhibited the slight residual proteolytic contamination. Neuraminidase was free of protelytic activity as determined by the lack of hydrolysis of azocasein (19). Lactoperoxidase, a gift from Dr. M. Morrison, was isolated from bovine milk by the method of Morrison and Hultquist (20); myosin was isolated from human platelets as described by Pollard et al. (21). Sodium dodecyl sulfate (Sigma Chemical Co.) was recrystallized from ethanol, and acrylamide (Eastman Organic Chemicals Div., Eastman Kodak Co., Rochester, N. Y.) from chloroform. All other chemicals were reagent grade.

Isolation of platelets. Unless otherwise indicated, 17.2 $\mathrm{ml}$ of blood was drawn from each normal donor and each thrombasthenic patient and mixed with $2.8 \mathrm{ml}$ of acid citrate dextrose (22). The blood was centrifuged immediately at $160 \mathrm{~g}$ for $10 \mathrm{~min}$ at room temperature to obtain plateletrich plasma. The platelets were then separated from plasma within $4 \mathrm{~h}$ after collection and washed as described by Massini and Lüscher (23). The buffer for the first washing step contained $0.12 \mathrm{M}$ sodium chloride, $0.0129 \mathrm{M}$ trisodium citrate, and $0.03 \mathrm{M}$ glucose; for the last step it contained $0.154 \mathrm{M}$ sodium chloride, $0.01 \mathrm{M}$ Tris, and $1 \mathrm{mM}$ EDTA, pH 7.4. The washed platelets were resuspended in the EDTA buffer at a concentration of $1 \times 10^{9}$ platelets $/ \mathrm{ml}$. More than $99.9 \%$ of the cells isolated by this procedure were platelets, as determined by phase-contrast microscopy.

Iodination of washed platelets. $1 \mathrm{mCi}$ of ${ }^{125} \mathrm{I}$ followed by $10 \mu \mathrm{l}$ of $0.1 \mathrm{M}$ sodium phosphate, $\mathrm{pH} 7.4$, containing $0.25 \mathrm{nmol}$ of lactoperoxidase, was added to stirred, washed $1-\mathrm{ml}$ platelet suspensions containing $1 \times 10^{9}$ platelets (13, 14). Sufficient iodination was obtained by adding to the platelet suspensions, at 10 -s intervals, five 10- $\mu$ l aliquots of freshly prepared $3 \mathrm{mM}$ hydrogen peroxide solution (1 $\mathrm{mM}$ EDTA buffer), followed by $9 \mathrm{ml}$ of the EDTA buffer. The platelets were sedimented by centrifugation, washed once with 10 $\mathrm{ml}$ of the same buffer and recentrifuged. After the buffer was completely removed, the centrifugation tube containing the platelet pellet was flushed with nitrogen gas. The platelets were solubilized by rapid suspension in $0.2 \mathrm{ml}$ of deaerated water, to which $0.1 \mathrm{ml}$ of deaerated $10 \%$ sodium dodecyl sulfate (SDS) was immediately added. The suspension was then immersed in boiling water for $3 \mathrm{~min}$ to complete solubilization. The solubilization step and subsequent incubations were performed in an atmosphere of nitrogen gas. The solubilized platelets were sealed under nitrogen gas in a glass ampoule.

Tritium labeling of washed platelets. Treatment of platelets with galactose oxidase followed by reduction with tritiated sodium borohydride, $\left[{ }^{3} \mathrm{H}\right] \mathrm{NaBH}_{4}$, permitted specific labeling of surface galactose and galactosamine residues in glycoproteins (24). Incubation of $1 \mathrm{ml}$ of washed platelets containing $1 \times 10^{9}$ platelets $/ \mathrm{ml}$ with $10 \mathrm{U}$ of Vibrio cholerae neuraminidase for $1 \mathrm{~h}$ in Tyrode's solution at $37^{\circ} \mathrm{C}$ was followed by incubation with $7.5 \mathrm{U}$ of galactose oxidase for $1.5 \mathrm{~h}$ at $37^{\circ} \mathrm{C}$. Then $2.5 \mathrm{mCi}$ of $\left[{ }^{3} \mathrm{H}\right] \mathrm{NaBH}_{4}$ was added and the mixture was incubated for $1 \mathrm{~h}$ at $37^{\circ} \mathrm{C}$. The cells were washed between each incubation step and then solubilized anaerobically as described for the iodinated samples and electrophoresed.

SDS-polyacrylamide gel electrophoresis. For one-dimensional gels, samples of labeled, SDS-solubilized platelets (10-20 $\mu \mathrm{l}$ containing $50 \mu \mathrm{g}$ of protein) were mixed with 50 $\mu l$ of sample buffer either in the presence or absence of reducing agents. Protein concentrations were determined by the method of Lowry et al. (25) with Moni-Trol as the standard. Nonreduced polypeptides were separated by incubating the samples at $100^{\circ} \mathrm{C}$ for $3 \mathrm{~min}$ with an anaerobic buffer free of reducing agents. To obtain complete reduction, the samples were heated at $100^{\circ} \mathrm{C}$ for $10 \mathrm{~min}$ with a buffer containing $10 \% \beta$-mercaptoethanol. The samples were electrophoresed in a commercial slab-gel apparatus (Model 220, Bio-Rad Laboratories, Richmond, Calif.) by the method of Laemmli (26). The resolving slab (16-cm width, 9-cm length, and $0.15-\mathrm{cm}$ thickness) contained $7.5 \%$ acrylamide and 
$0.1 \%$ SDS and was covered with a $1-\mathrm{cm}$ layer of $3 \%$ acrylamide stacking gel. The slot for each sample was $0.8-\mathrm{cm}$ wide. Electrophoresis was performed at $25 \mathrm{~V}$ for approximately $18 \mathrm{~h}$ (the time required for the tracking dye to reach the bottom of the gel). The polypeptides were stained with Coomassie Blue (27). Carbohydrate staining by the PAS procedure required a high concentration of protein; therefore, $400 \mu \mathrm{g}$ of protein was analyzed in cylindrical gels prepared as above and stained with PAS reagent (14). Molecular weights were estimated ${ }^{2}$ from comparisons with standard proteins: platelet myosin $(200,000 \mathrm{~mol} w \mathrm{wt}), \beta-$ galactosidase $(130,000 \mathrm{~mol} w \mathrm{w})$, bovine serum albumin $(68,000 \mathrm{~mol} \mathrm{wt})$, ovalbumin $(43,000 \mathrm{~mol} \mathrm{wt})$, and chymotrypsinogen A $(25,700 \mathrm{~mol} \mathrm{wt})$.

For nonreduced-reduced two-dimensional electrophoresis, approximately $50 \mu \mathrm{l}$ of iodinated, SDS-solubilized platelets containing $200 \mu \mathrm{g}$ of protein was mixed with $50 \mu \mathrm{l}$ of nonreducing sample buffer and electrophoresed in the same slab-gel apparatus as described above. The lane containing the resolved platelet polypeptides was trimmed to a width of $6 \mathrm{~mm}$ and incubated in $5 \mathrm{ml}$ of the reducing sample buffer containing $10 \% \beta$-mercaptoethanol for $15 \mathrm{~min}$ at $60^{\circ} \mathrm{C}$ to reduce the disulfide bonds of platelet polypeptides. The acrylamide strip was rinsed twice with the electrode buffer and placed on top of the stacking gel of the second slab. This sample was electrophoresed for $90 \mathrm{~min}$ at $15 \mathrm{~V}$ and then $25 \mathrm{~V}$ until the tracking dye reached the bottom of the gel. Protein staining and molecular-weight determinations were performed as described for one-dimensional gels.

Autoradiography. Stained gels of iodinated platelet samples were dried under vacuum and stored next to $\mathrm{X}$-ray film (Royal X-O-Mat RP/R-St., Eastman Kodak Co.). After 1-5 days of exposure, the film was processed as suggested by the manufacturer. Fluorograms of gels containing tritiated samples were prepared by the method of Bonner and Laskey (30). In one experiment (Table I), iodinated polypeptides were cut from the gels and the amount of radioactivity was determined with a gamma radiation counter.

Preparation of the Lens culinaris lectin affinity column. Lectin was isolated from the common lentil (Lens culinaris) by the procedure of Howard et al. (31). The final purification step consisted of adsorption on Sephadex G-100 (Pharmacia Fine Chemicals Inc., Piscataway, N. J.) and elution with $0.05 \mathrm{M}$ D-glucose. Analysis of the purified material by SDS disc-gel electrophoresis showed a single band. The lectin (60 mg protein) was dialyzed against $0.2 \mathrm{M}$ sodium bicarbonate, $\mathrm{pH} 9.5$, and added to an equal volume $(10 \mathrm{ml})$ of cyanogen bromide-activated Sepharose 4B (Pharmacia Fine Chemicals, Inc.) (32) in the presence of $0.1 \mathrm{mM} \mathrm{MnCl}_{2}$ and $2 \% \alpha$-methyl mannoside. The resulting slurry was stirred for $18 \mathrm{~h}$ at $4^{\circ} \mathrm{C}$, and after $0.75 \mathrm{~g}$ of glycine was added, was stirred an additional $2 \mathrm{~h}$. Unattached lectin was removed from the Sepharose beads by three washes with $10 \mathrm{vol}$ of $0.05 \mathrm{M}$ Tris, $\mathrm{pH}$ 7.6. The beads were isolated by centrifugation, suspended in $10 \mathrm{ml}$ of $1.2 \%$ sodium deoxycholate (DOC), poured into a glass column $(1.1 \times 8 \mathrm{~cm})$, and washed successively with $10 \mathrm{ml}$ of $1.2 \%$ DOC, $50 \mathrm{ml}$ of $2.5 \% \alpha-$ methyl mannoside in $1.2 \% \mathrm{DOC}$, and finally with $1.2 \%$ DOC.

Isolation of platelet receptors. Platelet plasma membranes

${ }^{2}$ Many of the membrane polypeptides in this study contained carbohydrate and disulfide bonds, two factors that can result in errors in determination of molecular weight by SDS-gel electrophoresis $(28,29)$. Consequently, the molecular weights for glycoproteins are apparent only. were prepared by the glycerol lysis method (33) from pooled platelet concentrates within $24 \mathrm{~h}$ of blood collection. Isolated platelet membranes were solubilized in $1.2 \%$ DOC and centrifuged at $100,000 \mathrm{~g}$ for $1 \mathrm{~h}$ (about $90 \%$ of the membrane protein was solubilized). The clear supernate, containing $2.5 \mathrm{mg}$ protein in $5 \mathrm{ml}$, was allowed to flow through the L. culinaris lectin column over a period of $30 \mathrm{~min}$. The column was washed with $50 \mathrm{ml} 1.2 \%$ DOC, until the optical density $\left(\mathrm{OD}_{280}\right)$ of the eluate reached background, and then with $50 \mathrm{ml}$ of $2.5 \% \alpha$-methyl mannoside in $1.2 \%$ DOC to elute material with receptors for $L$. culinaris lectin. Most of the proteins were not retained and flowed directly through the column. No further ultraviolet-absorbing material was eluted until $\alpha$-methyl mannoside was added. After the $\mathrm{OD}_{280}$ of each $2.0-\mathrm{ml}$ fraction was determined, the tubes containing the "flow-through" material and the $\alpha$-methyl mannoside-eluted peaks were pooled, dialyzed extensively against $\mathrm{H}_{2} \mathrm{O}$, lyophilized, solubilized in $2 \%$ SDS, and analyzed by electrophoresis.

\section{RESULTS}

Iodination of normal and thrombasthenic platelets. At least six glycoproteins, with molecular weights ranging from 97,000 to 170,000 , are normally exposed on the platelet membrane surface (13). When iodinated membrane polypeptides of normal platelets were separated by electrophoresis without reduction, and the distribution of radioactivity determined by autoradiography, five distinct bands, corresponding in electrophoretic mobilities to glycoproteins Ia, Ib, IIa, IIb, and III, were present (Fig. 1). An additional glycoprotein, IV, has a molecular weight similar to that of glycoprotein III but iodinates much less readily (13) and was therefore not detected by this technique. Comparison of the polypeptide composition of normal vs. thrombasthenic platelet samples indicated strikingly decreased iodination in regions corresponding to the electrophoretic mobilities of glycoproteins IIb and III on thrombasthenic platelets.

Nonreduced-reduced two-dimensional electrophoresis. Cleaving the disulfide bonds of platelet membrane glycoproteins has a profound effect on their electrophoretic mobilities (13). When the bonds that link glycoproteins to small subunits ( $\beta$-chains) are cleaved, the electrophoretic mobilities of the larger subunits ( $\alpha$-chains) are greater than those of the parent molecule. Conversely, cleavage of intramolecular disulfide bonds causes a decrease in electrophoretic mobility. Thus, glycoproteins can be more completely separated by nonreduced-reduced two-dimensional electrophoresis than by simple one-dimensional separation.

The iodinated polypeptides of normal and thrombasthenic platelets were separated by nonreducedreduced, two-dimensional electrophoresis. In the first step of the procedure, solubilized platelet polypeptides with intact disulfide bonds were separated by electrophoresis. Separation in the second dimension was 


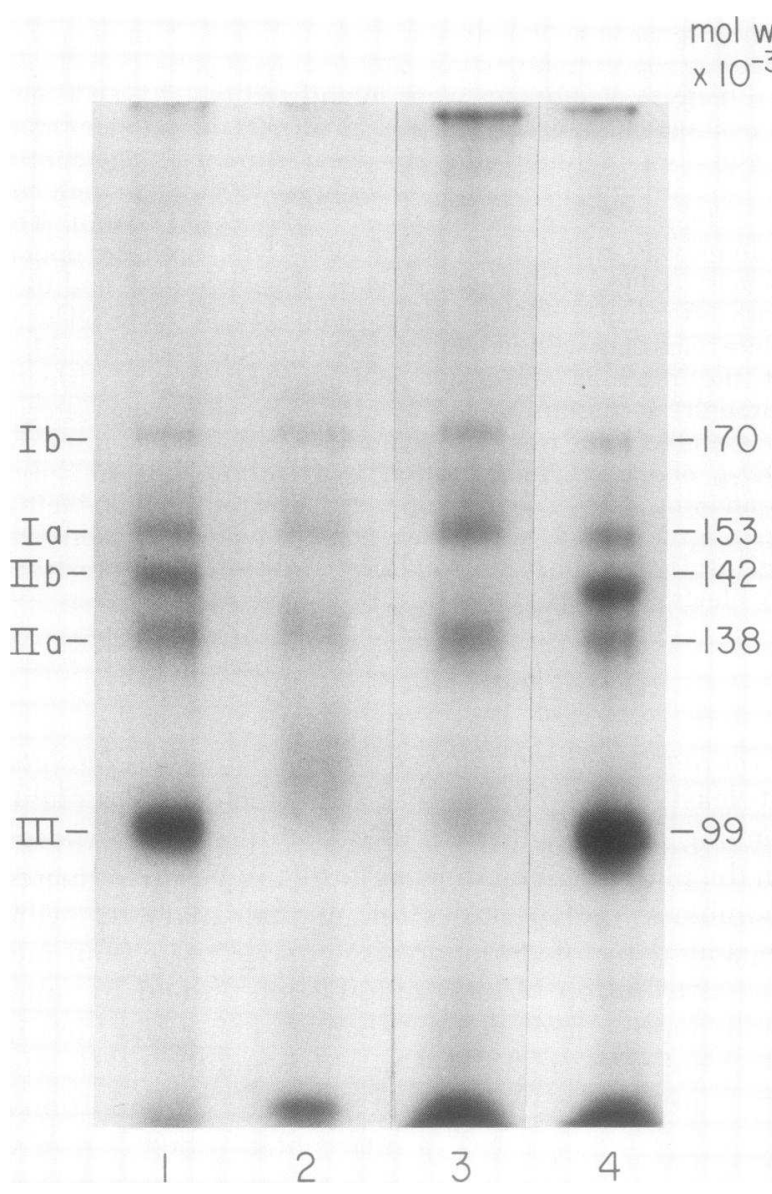

FIGURE 1 Autoradiogram of ${ }^{125}$ I-labeled polypeptides separated in acrylamide gels. Washed platelets from two thrombasthenic patients and two normal donors were labeled by lactoperoxidase-catalyzed iodination and solubilized anaerobically in SDS. The sample was electrophoresed with disulfide bonds intact (no reduction) directly on a $7.5 \%$ polyacrylamide slab gel, stained for protein, and dried. The figure is an autoradiogram of the dried gel. Normal controls are designated as 1 and 4 , and thrombasthenic samples as 2 and 3.

performed after the disulfides in the resolved polypeptides were cleaved with $10 \% \beta$-mercaptoethanol. With this two-dimensional electrophoretic procedure, any component not containing a disulfide linkage appeared on a diagonal line, since its mobility was the same in both directions. Any shift from the line indicated that the polypeptide contained a disulfide bond. In Fig. 2A, an autoradiogram of a normal sample, all six previously identified (13) glycoproteins are resolved. Only the $\alpha$-chains of the dimeric glycoproteins, Ib and IIb, are visible, since the $\beta$-chains migrate off the $7.5 \%$ acrylamide gel (13). We have not yet determined if the additional radioactive component present, I $\mathrm{C}_{\alpha}$, is also glycosylated. The decrease in electrophoretic mobility of glycoprotein III after reduc- tion with $\beta$-mercaptoethanol resulted in good separation of the molecule from glycoprotein IV, which was unaffected by reduction. The labeling pattern of reduced polypeptides from thrombasthenic platelets (Fig. 2B) showed two conspicuous differences from the pattern of normal reduced samples: there was greatly decreased iodination of $\mathrm{IIb}_{\alpha}$ and of III, now distinct from IV.

Quantitation of labeling differences. To quantitate the observed differences in iodination among major membrane polypeptides of thrombasthenic vs. normal platelets, we compared the amounts of radioactive iodine incorporated into the various glycoproteins. ${ }^{3}$ Compared in Table I are the mean amounts of radioactivity incorporated by glycoproteins $\mathrm{Ib}_{\alpha}, \mathrm{IIb}_{\alpha}$, and III, relative to that of Ia. In thrombasthenic samples, glycoproteins $\mathrm{IIb}_{\alpha}$ and III incorporated about 60 and $80 \%$ less radioactivity than the corresponding fractions of normal samples; incorporation by $\mathrm{Ib}_{\alpha}$ was essentially the same in both groups.

Tritium labeling of normal and thrombasthenic platelets. Lactoperoxidase-catalyzed iodination labels only cell-surface tyrosyl and histidyl residues and is not specific for glycoproteins (35). Another labeling technique, specific for carbohydrate residues, was therefore used as an alternate to iodination. This technique utilizes oxidation by galactose oxidase followed by reduction with tritiated sodium borohydride to specifically label surface galactose and galactosamine residues in glycolipid and glycoprotein (29). Labeling patterns of glycoproteins in thrombasthenic vs. normal platelets were determined after separation of polypeptides by gel electrophoresis. Comparison of autofluorograms of reduced samples (Fig. 3A) indicated the same decreased labeling of glycoproteins IIb $_{\alpha}$ and III in thrombasthenic platelets seen on autoradiograms of iodinated samples (Fig. 3C). In addition, the proteinstained gels (Fig. 3B) also showed decreased staining of a polypeptide corresponding in molecular weight to glycoprotein III. When an autofluorogram of the tritiated normal sample was slightly overexposed, all

\footnotetext{
${ }^{3}$ Absolute values for platelet samples could not be compared because the extent of iodination depends on a number of variables (e.g., the rate of hydrogen peroxide utilization by the platelet) and results often vary widely among subjects. However, since only a small percentage of each surface component is iodinated (34), the amount of label in each component is proportional to its concentration on the membrane surface. Accordingly, on a platelet sample from one individual, the amount of label incorporated by one platelet membrane glycoprotein, relative to that incorporated by another, is independent of the total amount of label incorporated. This is shown in Table I, where the ratios of glycoprotein iodination within either normal or thrombasthenic groups were independent of the amount of iodination. Various iodinations of human erythrocyte stroma have produced similar results (35).
} 

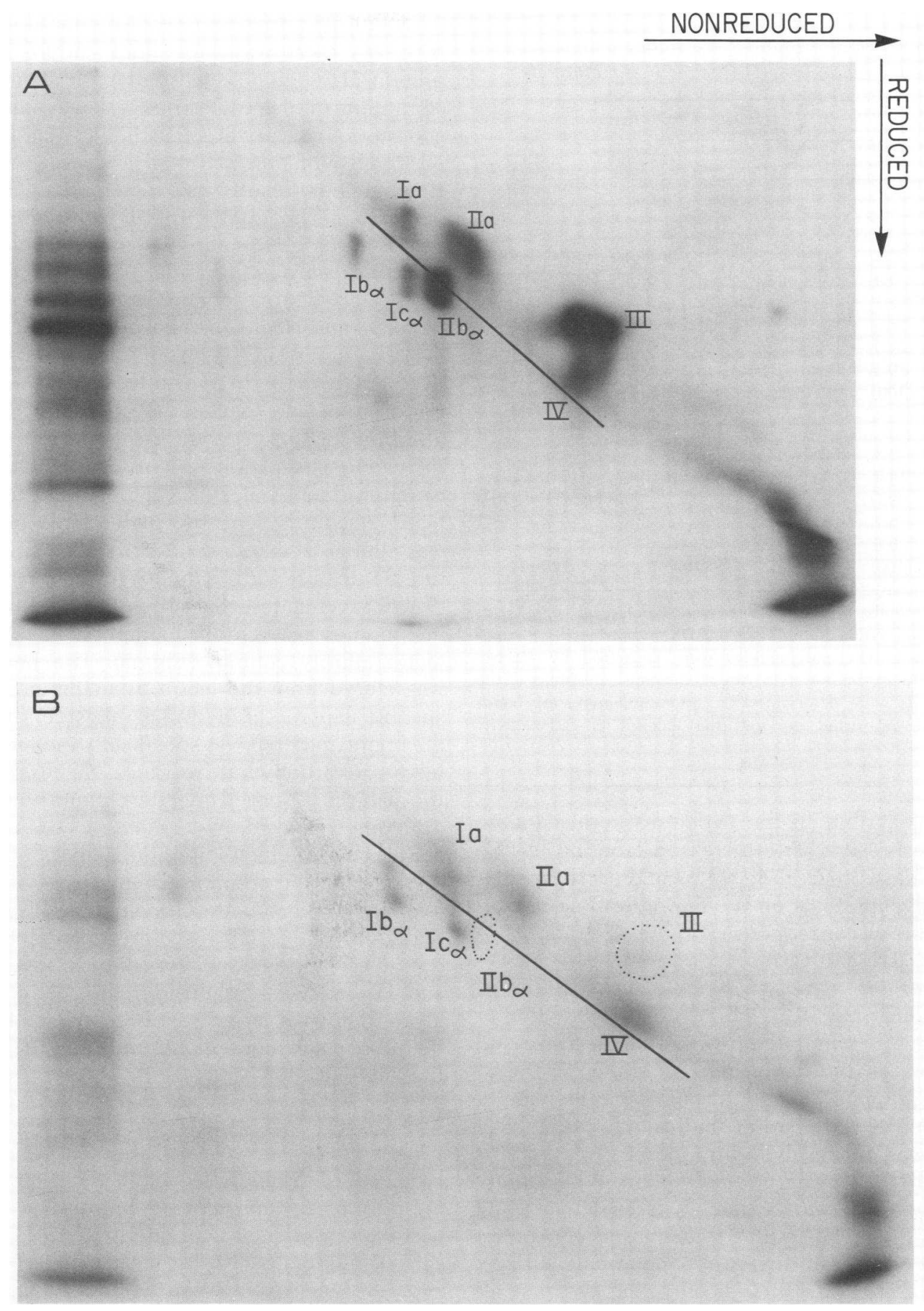

FIGURE 2 Separation of iodinated polypeptides of normal (A) and thrombasthenic (B) platelets by nonreduced-reduced two-dimensional electrophoresis. Thrombasthenic and normal platelets were washed, labeled by lactoperoxidase-catalyzed iodination, solubilized anaerobically in SDS, and subjected to two-dimensional analysis. The samples were first electrophoresed without reduction (disulfide bonds intact). Polypeptides resolved by this separation were then reduced with $10 \% \beta$-mercaptoethanol to cleave disulfide bonds and electrophoresed in the second dimension. The figures are autoradiograms of the dried gels. In $\mathrm{B}$, the dotted circles indicate the positions of $\mathrm{IIb}_{\alpha}$ and III in gels of normal samples. Slight labeling by $\mathrm{IIb}_{\alpha}$ and III could be seen if the autoradiograms were overexposed. The gel to the left of each diagonal is a one-dimensional separation of the reduced sample. 
TABLE I

Relative Distribution of Radioactive Iodide among Major Glycoprotein Fractions of Thrombasthenic Platelets*

\begin{tabular}{|c|c|c|c|c|}
\hline & \multicolumn{4}{|c|}{ Incorporation of ${ }^{125}$ I by fraction } \\
\hline & Ia & $\mathrm{Ib}_{\alpha}$ & $\mathrm{IIb}_{\alpha}$ & III \\
\hline & \multicolumn{4}{|c|}{$10^{3} \mathrm{cpm}=\%$ of $\mathrm{Ia}$} \\
\hline \multicolumn{5}{|l|}{ Control } \\
\hline 1 & 5.8 & $8.6=148 \%$ & $16.7=288 \%$ & $34.6=598 \%$ \\
\hline 2 & 9.0 & $10.1=112 \%$ & $22.9=255 \%$ & $58.5=651 \%$ \\
\hline 3 & 5.1 & $6.2=121 \%$ & $12.1=239 \%$ & $24.7=487 \%$ \\
\hline 4 & 11.5 & $11.3=98 \%$ & $16.2=140 \%$ & $36.7=318 \%$ \\
\hline 5 & 14.0 & $17.5=125 \%$ & $27.9=199 \%$ & $65.1=466 \%$ \\
\hline 6 & 10.4 & $16.4=159 \%$ & $27.1=262 \%$ & $60.0=569 \%$ \\
\hline Mean $\% \pm \mathrm{SD}$ & $(100)$ & $127 \pm 23$ & $230 \pm 53$ & $515 \pm 118$ \\
\hline \multicolumn{5}{|l|}{ Patient } \\
\hline 1 & 3.3 & $4.8=145 \%$ & $3.8=114 \%$ & $4.0=120 \%$ \\
\hline 2 & 14.9 & $16.2=109 \%$ & $16.2=109 \%$ & $15.0=100 \%$ \\
\hline 3 & 9.7 & $12.2=125 \%$ & $9.7=99 \%$ & $12.3=126 \%$ \\
\hline 4 & 8.7 & $10.5=121 \%$ & $10.3=118 \%$ & $10.9=125 \%$ \\
\hline 5 & 5.6 & $7.2=129 \%$ & $5.9=105 \%$ & $7.2=129 \%$ \\
\hline Mean $\% \pm \mathrm{SD}$ & $(100)$ & $126 \pm 13$ & $109 \pm 7$ & $120 \pm 12$ \\
\hline
\end{tabular}

* Normal and thrombasthenic platelets were labeled by lactoperoxidase-catalyzed iodination, solubilized in SDS and reduced with $10 \%$ $\beta$-mercaptoethanol. The polypeptides were separated by electrophoresis on $7.5 \%$ acrylamide gels. After the precise positions of the radioactive bands were determined by autoradiography, the bands were cut from the dried gel and the amount of radioactivity determined.

glycoproteins visible in the tritiated thrombasthenic sample were present. The increased labeling by the galactose oxidase procedure of some of the low molecular weight glycoproteins on the thrombasthenic platelets could be a consequence of the alterations in glycoproteins IIb and III.

Protein staining of polypeptides from normal and thrombasthenic platelets. The decreased labeling of glycoprotein IIb can be attributed to a decreased concentration of the molecule, as shown by PAS staining of polypeptides in thrombasthenic platelet membranes $(10,11)$. In earlier studies, however, glycoprotein III was not resolved from other glycoproteins by carbohydrate staining of electrophoresed, partially reduced samples $(10-13)$, so that its decreased labeling could be the result of either a decreased concentration or steric inaccessibility on the membrane surface. The results from Fig. 3B indicated that the first alternative was likely; i.e., that the concentration of glycoprotein III was lower in thrombasthenic platelets. This alternative was examined further by staining polyacrylamide gels for protein after the normal and thrombasthenic membrane polypeptides had been separated by nonreduced-reduced, two-dimensional electrophoresis. Without disulfide bonding, glycoprotein III decreased in electrophoretic mobility in normal samples and was clearly separated from other polypeptides (Fig. 4A). In thrombasthenic samples (Fig. 4B), there was only slight staining in the glycoprotein III region, consistent with a decreased concentration of the molecule. A lower amount of glycoprotein $\mathrm{IIb}_{\alpha}$ in thrombasthenic platelets was also observed with this staining procedure. Staining with PAS reagent (results not shown) likewise showed a decreased concentration of glycoproteins IIb and III in thrombasthenic samples.

Affinity chromatography. Some glycoproteins in the erythrocyte membrane aggregate in SDS and appear at more than one place on the acrylamide gel (36). To determine if glycoproteins IIb and III have different carbohydrate residues, and hence are discrete molecular species, we measured their relative affinities for $L$. culinaris lectin, which binds primarily to mannosyl and glucosyl residues in carbohydratecontaining molecules (37). The approach for isolating lectin receptors from the platelet plasma membrane was similar to that described by Hayman and Crumpton (38) for use with lymphocyte plasma membranes.

A deoxycholate extract from normal platelet plasma membranes was chromatographed on a $L$. culinaris lectin affinity column (Fig. 5). The bound material was eluted from the lectin by using an excess of the specific haptene, $\alpha$-methyl mannoside, indicating that the bound material was specifically interacting with the lectin via carbohydrate residues. No ultraviolet-absorbing material adsorbed onto the column when Sepharose 4B alone was used.

Fig. 6 is an electrophoretic analysis of the proteins eluted from the column. Comparisons of gels stained for protein (Fig. 6A) and carbohydrate (Fig. 6B) showed that glycoprotein IIb was almost completely adsorbed onto the lectin column. By contrast, glycoproteins III and IV had weak affinity for the lectin. In addition, glycoprotein Ib and a small amount of IIa were also bound and subsequently eluted from the column with the haptene.

\section{DISCUSSION}

We conclude from these results that there is a paucity not only of glycoprotein IIb but also of III in thrombasthenic platelets. Our interpretation conflicts with earlier judgments $(10,11)$ that glycoprotein III is unaltered. One explanation is that glycoproteins III and IV were not separated by previous electrophoretic techniques (10-12). Although, as isolated without reduction, the two components have similar apparent molecular weights $(99,000$ vs. 97,000$)$, they can be resolved after extensive reduction (13). The apparent molecular weight of III increases from 99,000 to 114,000 when its disulfide bonds are cleaved by reducing agents, while the electrophoretic mobility of IV appears to be unchanged. This suggests that glyco- 


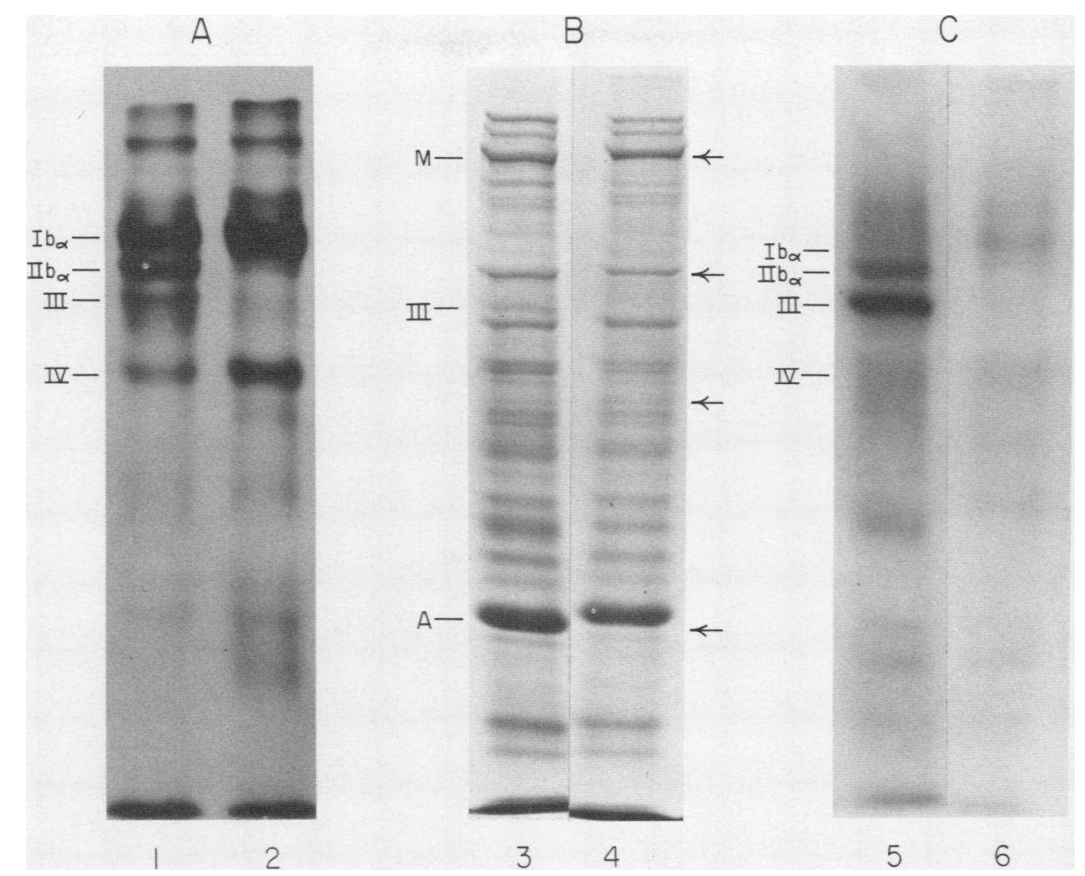

FIGURE 3 Comparison of galactose oxidase/ $\mathrm{Na}\left[{ }^{3} \mathrm{H}\right] \mathrm{BH}_{4}$ labeling and lactoperoxidase-catalyzed iodination of normal and thrombasthenic platelets. Platelets from a normal donor (gels 1,3 , and 5) and a thrombasthenic pateint (gels 2, 4, and 6) were labeled by either the galactose oxidase procedure or lactoperoxidase-catalyzed iodination. The polypeptides and glycoproteins were then solubilized in SDS, reduced, and electrophoresed on $7.5 \%$ polyacrylamide gels. The autofluorograms in A show the distribution of tritium in the gels of galactose oxidase $/ \mathrm{Na}\left[{ }^{3} \mathrm{H}\right] \mathrm{BH}_{4}$ labeled platelets. The polypeptides in $\mathrm{B}$ were stained for protein with Coomassie Blue. The autoradiograms in $\mathrm{C}$ show the distribution of iodine in the gels of lactoperoxidase-treated platelets. $M$ refers to platelet myosin, $A$ to platelet actin. The arrows $(\leftarrow)$ in gel 4 designate the positions of molecular weight standards myosin, $\beta$-galactosidase, bovine serum albumin, and ovalbumin, respectively.

protein III contains intrasubunit disulfide bonds and glycoprotein IV does not. In previous studies, which made use of partially reduced platelet samples (1012), this distinction could not be made and glycoprotein IV in thrombasthenic platelets was incorrectly assumed to be glycoprotein III.

There is ample reason to believe that glycoproteins IIb and III are distinct molecules. First, they have unique molecular weights (13): they appear at different positions on the acrylamide gel, and are not interconverted when incubated at varying temperatures and times. Second, their polypeptide chains differ, as shown by the reaction of the molecules to reducing agents (13). Unlike glycoprotein III, which decreases in electrophoretic mobility after reduction, glycoprotein IIb dissociates into subunits upon disulfide cleavage. Third, the two glycoproteins have different labeling characteristics: III is labeled more intensely by lactoperoxidase-catalyzed iodination and IIb more intensely by the galactose oxidase procedure. Finally, normal glycoprotein IIb and III were partially resolved by affinity chromatography with the lectin from L. culinaris. This demonstrates that the two glycoproteins have different lectin receptors.

The results of nonreduced-reduced two-dimensional electrophoresis (Figs. 2 and 4) strongly suggest that the molecular expression of the platelet membrane defect in thrombasthenia is quantitative. Glycoproteins IIb and III always appeared in the same positions in the gel as their normal counterparts, but in much lower concentration. Even though their molecular weights are unaltered, we cannot say with certainty that these glycoproteins are completely homologous with the normal components. This interpretation agrees with studies of the inherited brush-border membrane disease, in which sucrase-isomaltase proteins are decreased in concentration and do not appear to be present in an altered form (39).

The phenotypic expression of Glanzmann's thrombasthenia, inherited as an autosomal recessive trait, is well characterized $(1,2)$. Still unclear is the genetic basis for alteration of two different molecules in the disease. A decreased concentration of these membrane components with no observable change in the function 

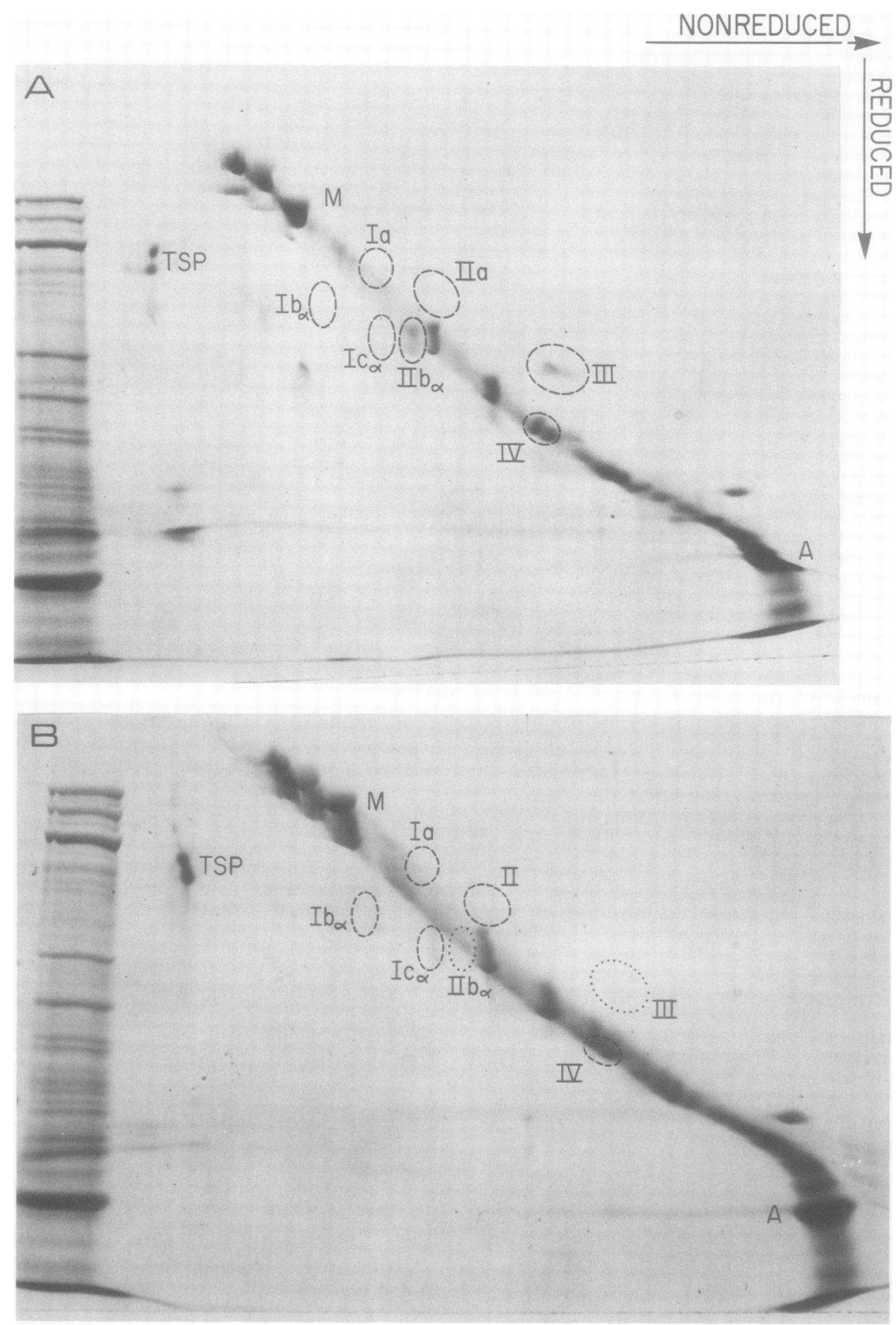

FIGURE 4 Protein staining of normal (A) and thrombasthenic (B) platelet polypeptides separated by nonreduced-reduced two-dimensional electrophoresis. Normal and thrombasthenic polypeptides were separated by the procedure described in Fig. 2 and stained with Coomassie Blue. The dashed circles indicate the positions of iodinated components in Fig. 2; the dotted circles in B correspond to the positions IIb and III in gels of normal samples. The gel to the left of each diagonal is a one-dimensional separation of the reduced sample.

of any other cell type suggests several possibilities. First, there could be a single mutation in a structural gene that specifies both glycoproteins. If so, then both components would be cleavage products of a larger precursor. Alternatively, the two polypeptides might be specified by different genes having linked expressions. Finally, there might be a defect in some platelet-specific post-translational process (e.g., glyco- 


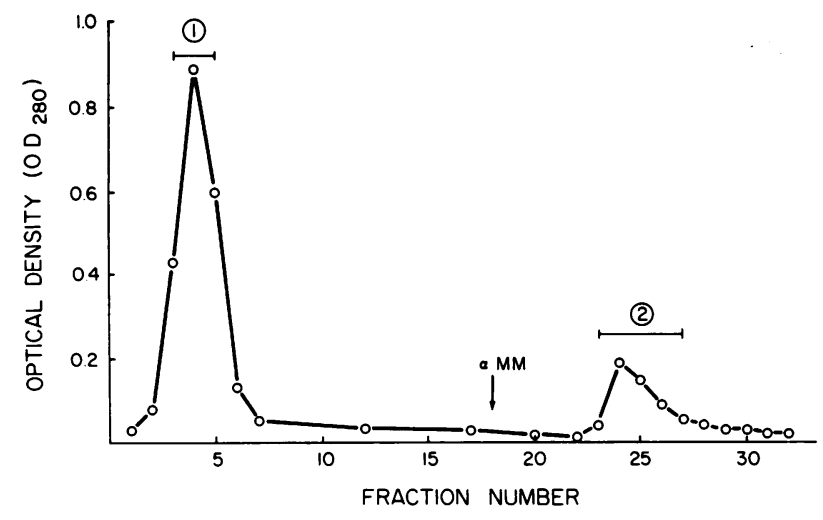

FIGURE 5 Affinity chromatography of DOC-solubilized plasma membranes of normal platelets. Platelet membranes were isolated by the glycerol lysis method and solubilized in $1.2 \%$ DOC. The solubilized membranes were chromatographed on a Sepharose B affinity column, containing L. culinaris lectin, as described in Methods. The haptene (2.5\% $\alpha$-methyl mannoside $[\alpha \mathrm{MM}]$ in $1.2 \% \mathrm{DOC})$ was added as indicated by the arrow. Peak 1 designates the "flow through", or membrane material not containing receptors for $L$. culinaris lectin; peak 2 designates the material eluted by the haptene. The bars indicate the fractions that were pooled.

sylation, proteolysis, phosphorylation) that is common to both glycoproteins. At present, it is not possible to distinguish between these possibilities. Determination of the protein composition of normal and thrombasthenic megakaryocytes may help in determining the genetic basis of the thrombasthenic phenotype.

Staining with Coomassie Blue appears to be the simplest method for screening thrombasthenic platelet samples for glycoprotein deficiencies. Protein staining on acrylamide gels requires only $50 \mu \mathrm{g}$ of protein, the amount contained in less than $0.5 \mathrm{ml}$ of blood, assuming a normal platelet count. Both of the altered glycoproteins contain disulfide bonds, however, so special precautions are required to assess their concentrations. For instance, glycoprotein IIb has a unique molecular weight, but after reduction, the subunits $\mathrm{IIb}_{\alpha}$ and $\mathrm{IIb}_{\boldsymbol{\beta}}$ co-electrophorese with other polypeptides on one-dimensional gels. Accordingly, only nonreduced samples should be used for evaluating the concentration of glycoprotein IIb by protein staining procedures. Glycoprotein III, by contrast, co-electrophoreses with other polypeptides in nonreduced gels, but can be readily identified in gels of reduced samples.

Several workers have postulated that many variants of thrombasthenia exist and that some heterogeneity of the biochemical defect can be anticipated $(2,40$, 41). However, of the eight patients (representing five different families) whose platelets have been examined by lactoperoxidase-catalyzed iodination, all have shown similar quantitative decreases in glycoproteins IIb and III. Platelets from patients with other inherited bleed- ing disorders, i.e., storage pool disease, von Willebrand's disease, Bernard-Soulier syndrome, and factor $\mathrm{V}$ deficiency, have also been examined by these techniques and did not have the same abnormalities that characterize thrombasthenic platelets. ${ }^{4}$ It seems likely, therefore, that the reduced concentration of glycoproteins IIb and III is limited to thrombasthenia. In a previous study (12), increased iodination of a component with an apparent molecular weight of approximately 150,000 was observed in the platelets from three thrombasthenic patients. In the present study, when iodinated samples were solubilized anaerobically and electrophoresed directly, no such component was detected. It is not possible at this time to say whether the previous result was an artifact of sample preparation (since anaerobic conditions for solubilization were not used) or if it was caused by a variant subclass in thrombasthenia.

Thrombasthenic platelets perform all the functions of normal platelets, except that of aggregation. The inability of these platelets to retract clots is no doubt linked to their inability to aggregate. Because the functional defect of these platelets is so limited, it seems probable that one or both of the deficient glycoproteins in these platelets is involved in platelet

${ }^{4}$ Phillips, D. R., C. S. P. Jenkins, M-J. Larrieu, and E. F. Lüscher. In preparation.
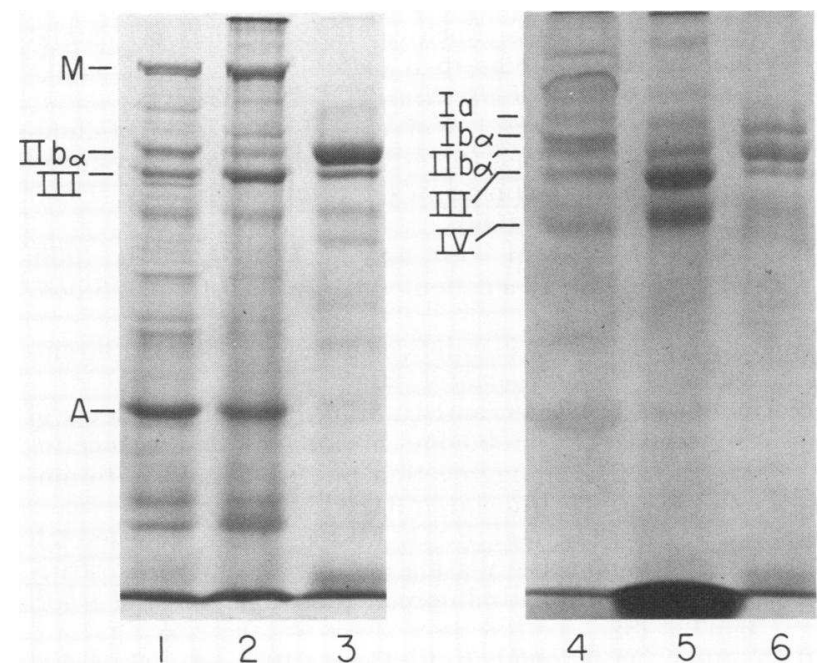

FIGURE 6 Electrophorograms of platelet glycoproteins containing receptors for $L$. culinaris lectin. Platelet membranes (gels 1 and 4) and materials forming peaks 1 (gels 2 and 5) and 2 (gels 3 and 6 ) in Fig. 5 were electrophoresed on $7.5 \%$ slab gels. Gels $1-3$ were stained for protein and gels 4-6 for carbohydrate. The gels contained $50 \mu \mathrm{g}$ protein $(1-3)$, $400 \mu \mathrm{g}$ protein (4 and 5 ), and $60 \mu \mathrm{g}$ protein (6). The slightly lower molecular weight of IIb in gels 3 and 6 was caused by a slight overloading of the gel to allow better visibility of impurities. 
aggregation. On the other hand, the receptor involved in aggregation might be a low molecular weight component that is not detected by presently available techniques. Further work on the functional role of membrane glycoproteins in aggregation is needed to resolve this important question.

Note added in proof. In recent experiments we have used a modified procedure for the galactose oxidase labeling and have obtained identical results (42).

\section{ACKNOWLEDGMENTS}

We would like to thank Doctors J. Gerrard, J. White (Minneapolis, Minn.), and G. White (Chapel Hill, N. C.) for graciously allowing us to study their patients and willingly giving their assistance. We would also like to thank Mrs. Linda Coats for her capable assistance with the L. culinaris work, Mr. John Gilbert for his help in the preparation of this manuscript, and Doctors M. Morrison and D. Kingsbury for their criticisms.

This work was supported by grant HL-15616 from the U. S. Public Health Service, National Institutes of Health.

\section{REFERENCES}

1. Hardisty, R. M., K. M. Dormandy, and R. A. Hutton. 1964. Thrombasthenia. Studies on three cases. Br.J. Haematol. 10: 371-387.

2. Caen, J. P., P. A. Castaldi, J. C. Leclerc, S. Inceman, M-J. Larrieu, M. Probst, and J. Bernard. 1966. Congenital bleeding disorders with long bleeding time and normal platelet count. Part I. Glanzmann's thrombasthenia (report of fifteen patients). Am.J. Med. 41: 4-26.

3. Stuart, M. J. 1975. Inherited defects in platelet function. Semin. Hematol. 12: 233-253.

4. Weiss, H. J., and S. Kochwa. 1968. Studies of platelet function and proteins in 3 patients with Glanzmann's thrombastheina. J. Lab. Clin. Med. 71: 153-165.

5. Karpatkin, S., and H. J. Weiss. 1972. Deficiency of glutathione peroxidase associated with high levels of reduced glutathione in Glanzmann's thrombasthenia. N. Engl. J. Med. 287: 1062-1066.

6. Zucker, M. B., J. H. Pert, and M. W. Hilgartner. 1966. Platelet function in a patient with thrombasthenia. Blood. 28: 524-534.

7. Walsh, P. N. 1972. Platelet coagulant activities in thrombasthenia. Br. J. Haematol. 23: 553-569.

8. Cohen, I., T. Glaser, and U. Seligsohn. 1975. Effects of ADP and ATP on bovine fibrinogen- and ristocetininduced platelet aggregation in Glanzmann's thrombasthenia. Br. J. Haematol. 31: 343-348.

9. Reddick, R. L., and R. G. Mason. 1973. Freeze-etch observations on the plasma membrane and other structures of normal and abnormal platelets. Am. J. Pathol. 70: $473-482$.

10. Nurden, A. T., and J. P. Caen. 1974. An abnormal platelet glycoprotein pattern in three cases of Glanzmann's thrombasthenia. Br. J. Haematol. 28: 233-253.

11. Nurden, A. T., and J. P. Caen. 1975. Specific roles for platelet surface glycoproteins in platelet function. Nature (Lond.). 255: 720-722.

12. Phillips, D. R., C. S. P. Jenkins, E. F. Lüscher, and M-J. Larrieu. 1975. Molecular differences of exposed surface proteins on thrombasthenic platelet plasma membranes. Nature (Lond.). 257: 599-560.
13. Phillips, D. R., and P. P. Agin. 1977. Platelet plasma membrane glycoproteins. Evidence for the presence of non-equivalent disulfide bonds using nonreduced-reduced two-dimensional gel electrophoresis. J. Biol. Chem. 252: 2121-2126.

14. Phillips, D. R. 1972. Effect of trypsin on the exposed polypeptides and glycoproteins in the human platelet membrane. Biochemistry. 11: 4582-4588.

15. White, J. G., E. Yunis, M. Colliander, and W. Krivit. 1964. The correction of prolonged bleeding times in two brothers by platelet transfusions despite normal in vitro platelet tests. Pediatrics. 33: 579-584.

16. White, J. G. 1972. Effects of cationic polypeptides on thrombasthenic and afibrinogenemic blood platelets. $\mathrm{Am}$. J. Pathol. 68: 447-458.

17. Gerrard, J. M., and J. G. White, 1967. The influence of aspirin and indomethacin on the platelet contractile wave. Am. J. Pathol. 82: 513-521.

18. Hatton, M. W. C., and E. Regoeczi. 1976. The proteolytic nature of commercial samples of galactose oxidase. Purification of the enzyme by a simple affinity method. Biochim. Biophys. Acta. 438: 339-346.

19. Dayton, W. R., D. E. Goll, M. G. Zeece, R. M. Robson, and W. J. Reville. 1976. A Ca ${ }^{++}$-activated protease possibly involved in myofibrillar protein turnover. Purification from porcine muscle. Biochemistry. 15: 2150-2158.

20. Morrison, M., and D. E. Hultquist. 1963. Lactoperoxidase. II. Isolation. J. Biol. Chem. 238: 2843-2849.

21. Pollard, T. D., S. M. Thomas, and R. Niederman. 1974. Human platelet myosin. I. Purification by a rapid method applicable to other nonmuscle cells. Anal. Biochem. 60: $258-266$.

22. Aster, R. M., and J. H. Jandl. 1964. Platelet sequestration in man. I. Methods. J. Clin. Invest. 43: 843-855.

23. Massini, P., and E. F. Lüscher. 1974. Some effects of ionophores for divalent cations on blood platelets. Comparison with the effects of thrombin. Biochim. Biophys. Acta. 372: 109-121.

24. Gahmberg, C. G., and S. I. Hakomori. 1973. External labeling of cell surface galactose and galactosamine in glycolipid and glycoprotein of human erythrocytes. $J$. Biol. Chem. 248: 4311-4317.

25. Lowry, O. H., N. J. Rosebrough, A. L. Farr, and R. J. Randall. 1951. Protein measurement with the Folin phenol reagent. J. Biol. Chem. 193: 265-275.

26. Laemmli, V. K. 1970. Cleavage of structural proteins during the assembly of the head of bacteriophage T4. Nature (Lond.). 227: 680-685.

27. Fairbanks, G., T. L. Steck, and D. F. H. Wallach. 1971. Electrophoretic analysis of the major polypeptides of the human erythrocyte membrane. Biochemistry. 10: $2606-2617$.

28. Fish, W. W., J. A. Reynolds, and C. Tanford. 1970. Gel chromatography of proteins in denaturing solvents. Comparison between sodium dodecyl sulfate and guanidine hydrochloride as denaturants. J. Biol. Chem. 245: 51665168.

29. Segrest, J. P., R. L. Jackson, E. P. Andres, and V. T. Marchesi. 1971. Human erythrocyte membrane glycoprotein: A reevaluation of the molecular weight as determined by SDS polyacrylamide gel electrophoresis. Biochem. Biophys. Res. Commun. 44: 390-395.

30. Bonner, W. A., and R. A. Laskey. 1974. A film detection method for tritium-labeled proteins and nucleic acids in polyacrylamide gels. Eur. J. Biochem. 46: 83-88.

31. Howard, I. K., H. J. Sage, M. D. Stein, N. M. Young, 
M. A. Leon, and D. F. Dyckes. 1971. Studies on a phytohemagglutin from the lentil. J. Biol. Chem. 246: $1590-1595$.

32. March, S. C., I. Parikh, and P. Cuatrecasas. 1974. A simplified method for cyanogen bromide activation of agarose for affinity chromatography. Anal. Biochem. 60: 149-152.

33. Barber, A. J., and G. A. Jamieson. 1970. Isolation and characterization of plasma membranes from human blood platelets. J. Biol. Chem. 245: 6357-6365.

34. Phillips, D. R., and M. Morrison. 1973. Changes in accessibility of plasma membrane protein as the result of tryptic hydrolysis. Nat. New Biol. 242: 213-215.

35. Phillips, D. R., and M. Morrison. 1971. Exposed protein on the intact erythrocyte. Biochemistry. 10: 1766-1771.

36. Tuech, J. K., and M. Morrison. 1974. Human erythrocyte membrane sialoglycoproteins: A study of interconversion. Biochem. Biophys. Res. Commun. 59: 352-360.

37. Sharon, N., and H. Lis. 1972. Lectins: Cell agglutina- bility and sugar specific problems. Science (Wash. D. C.). 177: 949-959.

38. Hayman, M. J., and M. J. Crumpton. 1972. Isolation of glycoproteins from pig lymphocyte plasma membrane using Lens culinaris phytohemagglutinin. Biochem. Biophys. Res. Commun. 47: 923-930.

39. Preiser, H., D. Menard, R. K. Crane, and J. J. Cerda. 1974. Deletion of enzyme protein from the brushborder membrane in sucrase-isomaltase deficiency. Biochim. Biophys. Acta. 363: 279-282.

40. Reichert, N., U. Seligsohn, and B. Ramot. 1975. Clinical and genetic aspects of Glanzmann's thrombasthenia in Israel. Report of 22 cases. Thromb. Diath. Haemorrh. 34: 806-820.

41. Weiss, H. J. 1975. Platelet physiology and abnormalities of platelet function. N. Engl. J. Med. 293: 580-588.

42. Phillips, D. R., and P. P. Agin. 1977. Platelet plasma membrane glycoproteins. Identification of a proteolytic substrate for thrombin. Biochem. Biophys. Res. Commun. 75: $940-947$. 